\title{
NUEVO REGISTRO DE LA NUTRIA MARINA Lontra felina (MOLINA, 1782) AL NORTE DE SU DISTRIBUCIÓN ACTUAL
}

\section{NEW RECORD OF THE MARINE OTTER Lontra felina (MOLINA, 1782) NORTH TO ITS CURRENT DISTRIBUTION}

\author{
Joanna Alfaro-Shigueto ${ }^{1,2}$, Juan Valqui ${ }^{34}$, Jeffrey C. Mangel $^{1,2}$
}

\begin{abstract}
Resumen
La nutria marina Lontra felina es una especie en peligro de extinción cuyo rango de distribución es controversial, siendo considerada el área de Chimbote $\left(9^{\circ} 10^{\prime} \mathrm{S}\right)$ como el límite norte de su distribución actual. Aquí se documenta por primera vez su presencia a $115 \mathrm{~km}$ al norte de Chimbote, en el puerto artesanal de Huanchaco $\left(08^{\circ} 04^{\prime} \mathrm{S}\right)$. La discusión de esta nota científica busca promover la generación de investigaciones que esclarezcan tanto los factores que determinan la presencia de la nutria marina en un determinado lugar, así como su rango de distribución límite norte. Se sugieren medidas de conservación a favor de la nutria marina y del hábitat costero a nivel local (puertos y ciudades) y regional (departamentos y países), aprovechando el carácter de especie bandera de L. felina.
\end{abstract}

Palabras clave: nutria marina, Lontra felina, norte del Perú, distribución, hábitat.

\begin{abstract}
The marine otter Lontra felina is an endangered species and uncertainty exists regarding the limits of its current range, which is considered to be Chimbote $\left(9^{\circ} 10^{\prime} \mathrm{S}\right)$. Here we present the first documented evidence of the species' presence $115 \mathrm{~km}$ to the north of Chimbote, in the artisanal port of Huanchaco $\left(8^{\circ} 04^{\prime} \mathrm{S}\right)$. The note seeks to promote the generation of research to help clarify the factors that determine the presence of marine otters in a given location and which determine the northern limit to their range. Local (fishing ports and towns) and regional (provinces and countries) conservation measures safeguarding the species and the coastal marine habitat are recommended and should take advantage of the flagship species character of L. felina.
\end{abstract}

Key words: marine otter, Lontra felina, distribution, northern Peru, habitat.

\section{Introducción.}

La nutria marina Lontra felina conocida en el Perú también como "gato marino" o "chungungo" es el mamífero marino más pequeño del mundo y habita el litoral rocoso del Océano Pacífico Oriental de Sudamérica. Mientras que hasta fines del Siglo XX sufrió los efectos de una caza intensiva (Sielfeld \& Castilla, 1999), en el presente existen diversas actividades humanas que afectan a la especie: la contaminación por la minería, la industria pesquera y los desagües; el desplazamiento de individuos de $L$. felina por especies introducidas (perros, gatos y ratas) que acompañan el proceso de urbanización; la caza dirigida y la captura incidental; la pesca con dinamita; entre otras (Majluf et al., 2002; Apaza et al., 2004; Mangel \& Alfaro-Shigueto, 2008; Medina-Vogel et al., 2008; Vianna et al., 2010; Mangel et al., 2011; Valqui, 2011). Debido al creciente impacto que estas actividades tienen en el ecosistema marino costero, esta especie se encuentra categorizada como "En Peligro” por la Unión Internacional para la Conservación de la Naturaleza (IUCN, 2011) y está también protegida por la legislación peruana (Decreto
Supremo DS-034-2004). Debido a la falta de constancia en los registros históricos sobre la especie en el último siglo existe controversia en cuanto al rango histórico de distribución de L. felina. En el sur, la especie habría llegado hasta el Cabo de Hornos (5559’S), en el sur de Chile (Brack, 1978; Castilla \& Bahamondes, 1979) y la Islas de los Estados (54²8'S), en el sur de Argentina (Larivière, 1998). Hoy en día Lontra felina ha sido declarada casi extinta en el territorio argentino (Cassini, 2008), mientras Sielfeld (1997) registra a la especie en la Tierra del Fuego, Chile. En el norte, la distribución de la especie habría llegado hasta la Isla Lobos de Tierra (6²5'S) Región Lambayeque, Perú, lo que fue documentado por Schweigger (1964). Debido al impacto antropogénico, en las últimas décadas el rango de distribución en la costa norte de Perú habría sufrido una reducción aproximada de 400 kilómetros, desde la Isla Lobos de Tierra hasta Chimbote $\left(9^{\circ} 10^{\prime} \mathrm{S}\right)$, Región Ancash, Perú, lugar que se fija como el límite norte de la distribución actual (Apaza et al., 2004; Sánchez \& Ayala, 2006; Valqui et al., 2010, Valqui, 2011) (Figura 1). 


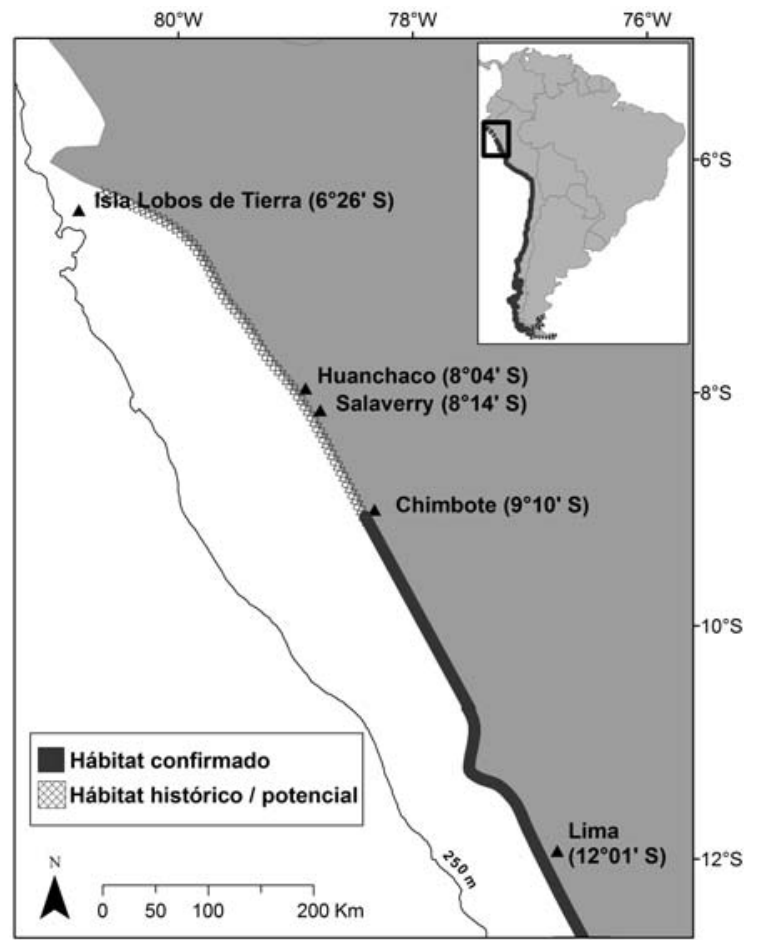

Figura 1. Mapa del área de estudio y límite norte de la distribución de la nutria marina, dentro del rango total de la especie por información histórica (línea punteada), así como por registros confirmados (línea continua). No se incluye la latitud en el mapa.

Los objetivos de esta nota son: (i) documentar los avistamientos de L. felina en el puerto de Huanchaco (804`S), Región La Libertad, Perú, (ii) sondear la percepción de los pobladores locales hacia la especie, (iii) motivar la discusión y la realización de posteriores investigaciones que determinen los factores ecológicos relevantes para la selección de hábitat de esta especie y (iv) contribuir a esclarecer su límite norte de distribución. Asimismo se discute la promoción de medidas de conservación aprovechando el carácter de especie bandera de L. felina.

Tabla 1. Preguntas hechas a los pescadores en puerto de Huanchaco. (A) respuesta abierta, (B) respuesta cerrada (sí o no).

1.¿Ha visto alguna vez una nutria marina en este puerto? (B)

2. ¿Cuántas nutrias marinas ha visto? (A)

3. ¿Las ve todo el año? (B)

4. ¿Qué impresión tiene del animal? (A)

5. ¿La nutria marina es un problema para Ud.? (A)

6. ¿Sabe si la especie está protegida por ley? (B)

\section{Materiales y métodos.}

En el marco de un proyecto de conservación de fauna marina, el 28 de noviembre del año 2009 llegó a los autores la comunicación personal de un pescador artesanal, que reportaba la presencia de nutrias marinas en el puerto de Huanchaco $\left(8^{\circ} 04^{\prime} \mathrm{S}\right)$. Dicho puerto se encuentra $115 \mathrm{~km}$ al norte del límite de distribución actual de la nutria marina: el puerto de Chimbote. Para corroborar la información, del 4 al 6 de diciembre de 2009 se realizaron observaciones entre las 7 y las 11 am en el muelle artesanal de Huanchaco. Adicionalmente, se realizaron encuestas de respuestas abiertas (en las cuales la respuesta es libre y el entrevistado puede explayarse) y cerradas (sí o no) (White et al., 2005) (Tabla 1). Se entrevistó a seis (06) pescadores en el muelle artesanal, mostrando paralelamente imágenes de la nutria marina para su reconocimiento. Se indagó sobre la presencia, abundancia y estacionalidad de la especie en el lugar, la opinión personal sobre el mamífero marino y el conocimiento de su protección legal. Entre Huanchaco y Chimbote no habita otra especie de nutria (Lontra longicaudis se encuentra $400 \mathrm{~km}$ más al norte y no tiene hábitos marinos), por lo que se puede descartar la posibilidad de confusión en el registro de la especie.

\section{Resultados.}

Durante las observaciones realizadas por los autores entre el 4 y el 6 de diciembre de 2009 se observó un (01) individuo moviéndose cerca del muelle artesanal (Figura 2). Durante los tres días de observación la nutria marina se refugió repetidamente debajo de la estructura del muelle, la cual presentaba restos de basura y desperdicios (Figura 3). En las entrevistas, cinco de los seis pescadores respondieron que han observado nutrias en Huanchaco alguna vez. Los mismos declararon haber visto solo un individuo sin que su presencia sea permanente a lo largo del año. Dos de aquellos pescadores detallaron que sus apariciones se limitan al verano. Cuatro de los seis pescadores entrevistados manifestaron su simpatía hacia las nutrias marinas, declarándolo un animal "bonito" al que no consideran un agente de

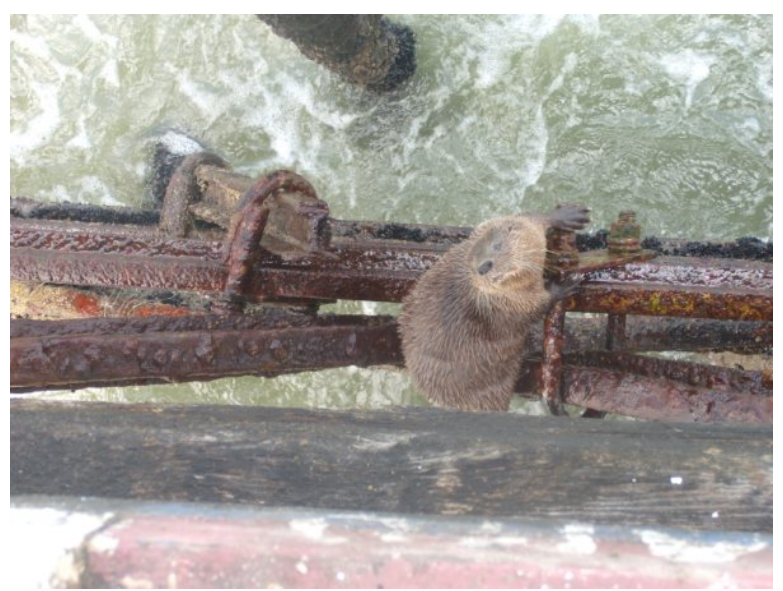

Figura 2. L. felina usando las estructuras del muelle artesanal de Huanchaco. 
competencia con su pesca. Es sabido que otra especie de mamífero marino, el lobo marino Otaria flavescens (Shaw, 1800), es visto como enemigo y fuerte competencia por los pescadores (Arias-Schreiber, 1993). Por otro lado, solo dos de los seis entrevistados sabían que la especie está protegida legalmente.

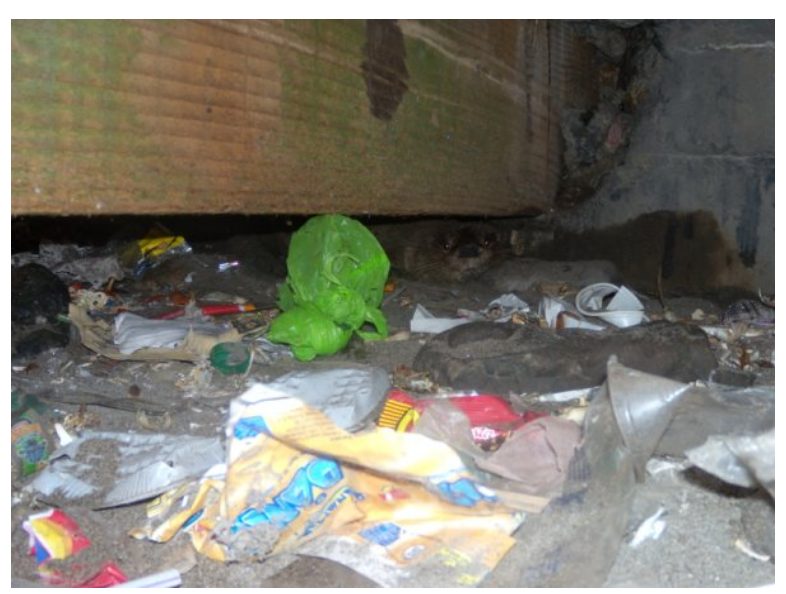

Figura 3. L. felina descansando bajo el muelle de Huanchaco, entre la basura.

\section{Discusión.}

El litoral del Pacífico sureste consta de zonas de litoral rocoso con hábitat apropiado para las nutrias marinas, las cuales necesitan de refugios seguros para establecerse en un lugar determinado (Sielfeld \& Castilla, 1999; Medina-Vogel et al., 2006). El litoral rocoso se alterna con zonas de playas desérticas no habitadas por la especie (Sielfeld \& Castilla, 1999). Medina-Vogel et al. (2008) postulan que esta fragmentación es un factor determinante en la distribución de nutrias marinas. De los $400 \mathrm{~km}$ entre la Isla Lobos de Tierra (6 $\left.6^{\circ} 25^{\prime} \mathrm{S}\right)$ y Chimbote $\left(9^{\circ} 10^{\prime} \mathrm{S}\right)$, la distancia entre parches rocosos promedia los 25,1 km, mientras que de los siguientes $400 \mathrm{~km}$, entre Chimbote $\left(9^{\circ} 10^{\prime} \mathrm{S}\right)$ y Ancón $\left(11^{\circ} 46^{\prime} \mathrm{S}\right)$, la distancia entre parches rocosos promedia 6,5 km (datos basados en calculos de los autores y en los datos de Valqui, 2011). Es posible que la baja disponibilidad de parches rocosos y la larga distancia entre ellos, en esta región de amplias playas de arena al norte de Chimbote, hayan presentado condiciones con elevado grado de adversidad para que las nutrias marinas establezcan su hábitat. Aunque hay casos conocidos de muerte por enmalle o por ingestión de restos para otros mamíferos (Thiel et al., 2010), no está claro en qué medida el grado de contaminación por basura en la zona (principalmente residuos plásticos, Figura 3) puede haber afectado la calidad de hábitat para las nutrias marinas. La contaminación por restos de basura (Thiel et al., 2010), o en todo caso, los efectos de la actividad antropogénica en general (la contaminación minera y pesquera, el desplazamiento por especies introducidas (perros, gatos y ratas), la caza dirigida, la captura incidental y la pesca con dinamita) son un problema a lo largo de toda la costa del Pacífico Oriental de Sudamérica (Majluf et al., 2002; Apaza et al., 2004; Mangel \& Alfaro-Shigueto, 2008; Medina-Vogel et al., 2008; Vianna et al., 2010; Mangel et al., 2011; Valqui, 2011). Las mencionadas condiciones sumadas a una incrementada mortalidad de L. felina durante eventos de "El Niño" (Apaza et al., 2004; Vianna et al., 2010) podrían haber actuado como las condicionantes de la desaparición de la especie en la región en las últimas décadas. A parte de un avistamiento eventual en el Puerto de Salaverry (8¹4’S) (Santillán \& Caro, 2007) en los últimos cuarenta años no se ha registrado la presencia de la especie entre las localidades de Isla Lobos de Tierra y Chimbote (Apaza et al., 2004; Sánchez \& Ayala, 2006; Valqui et al., 2010). La presencia de la especie en Huanchaco podría representar un evento de recolonización, lo que significa que la nutria marina habría superado barreras geográficas (largas playas de arena), pudiendo utilizar estructuras de muelles artesanales, espigones rompeolas y barcos varados como refugios temporales (Apaza et al., 2004; Medina-Vogel et al., 2008; Mangel et al., 2011) para llegar a las localidades abandonadas en el pasado. Incluso podría utilizarlas como refugios permanentes.

A diferencia de la situación descrita para el norte del Perú, las condiciones en los límites del sur del rango de distribución (Tierra del Fuego) de Lontra felina son distintas. Por un lado la intensidad de los efectos del fenómeno de "El Nino" (Grimm et al., 2000) es menor y la densidad poblacional humana en esta región austral es relativamente baja: 0,1 habitantes $/ \mathrm{km}^{2}$ (INE, 2011), a comparación de densidades entre 25 y 80 habitantes $/ \mathrm{km}^{2}$ para el litoral del norte peruano (INEI, 2007). Por otro lado, en la parte sur de sudamérica existe una estacionalidad más marcada (Luebert \& Pliscoff, 2006), lo que implicaría una variación en la oferta de alimento para L felina. Existe cierta controversia por las distintas opiniones de los autores sobre la ocurrencia de la especie en la Tierra del Fuego (Anderson et al., 2006; Chéhebar, 1990), mientras Vianna et al. (2010) sugieren la ausencia de la nutria marina en largos trechos de hasta $400 \mathrm{~km}$ a lo largo de todo el litoral chileno. Cassini (2008) y Vianna et al. (2010) destacan esta falta de información y la necesidad de profundizar los estudios, especialmente en las regiones más sureñas de Chile y Argentina.

Para aclarar la situación de la distribución a lo largo de todo el rango de distribución de Lontra felina, es necesario un mejor entendimiento de la ecología de la especie. Por ello se deben estudiar los factores que determinan un lugar del litoral como hábitat permanente de la nutria marina. Además, deben realizarse monitoreos periódicos a mediano y largo plazo para asegurar y documentar la presencia de la 
especie y esclarecer su rango de distribución tanto por el norte como por el sur. Tal como lo postulan Mangel et al. (2011) se debe monitorear y manejar las estructuras antropogénicas como refugios temporales o escalones ("stepping stones") en zonas de litoral normalmente no habitados por la especie. La misma función la podría cumplir un sistema de pequeños refugios artificiales. Por otro lado se sugiere un mayor control en el cumplimiento de la ley que prohibe la caza y comercialización de la nutria marina. Las medidas deben trascender el nivel local, ampliando su rango de acción a una escala regional (Perú, Chile y Argentina).

En la actualidad los pescadores son uno de los principales actores en las actividades de la costa peruana. En el futuro, lo serán los que hoy son escolares en puertos pesqueros y localidades de la costa. Por ello, se deben realizar campañas de educación, concientización y acción ambiental e involucrarlos en las medidas de conservación de la nutria marina en el Perú. Como se logró detectar en las entrevistas y por la experiencia de los autores en actividades de educación ambiental, la mayoría de personas sienten simpatía hacia la nutria marina, aunque muchos no saben que la especie está en peligro de extinción y que tiene un estatus de protección legal. El potencial que tiene L felina como especie bandera ha causado que algunos planes de conservación a nivel regional estén empezando a considerarla como especie emblemática de áreas marino costeras (Ulloa et al., 2010). Esta característica puede ser aprovechada en medidas de conservación en todo el litoral peruano, en especial en lo que se refiere al impacto de la pesquería artesanal y su interacción con la fauna marino costera.

\section{Conclusiones.}

Este artículo constituye la primera documentación de las últimas décadas de la nutria marina en Huanchaco, Perú, a 115 km del límite norte del rango de distribución actual. La presencia y los registros controversiales de L. felina en el litoral norte del Perú deben motivar los estudios sobre su selección de hábitat, la calidad de éste, así como el rol de estructuras de origen antropogénico y la distribución de la especie. A su vez se recomienda monitorear el impacto de las actividades humanas en el ecosistema marino costero, al mismo tiempo que se desarrollen medidas de conservación a nivel local y regional, aprovechando el carácter de especie símbolo de la nutria marina.

\section{Agradecimientos.}

Los autores agradecen a los pescadores locales que brindaron sus declaraciones en entrevistas y también a Sara Campos y Celia Cáceres por su colaboración. Las observaciones iniciales se realizaron en el marco del proyecto "Marine otter conservation project" a cargo de la organización Pro Delphinus. Los proyectos de investigación de los autores reciben el financiamiento de Columbus Zoo, People's Trust for Endangered Species, Darwin Sustainable Artisanal Fisheries Initiative - Peru, Rufford Small Grants y Yaqu Pacha. Los autores también agradecen la colaboración de dos árbitros anónimos, cuyas sugerencias contribuyeron significativamente en la elaboración de la versión final de este manuscrito.

\section{Literatura citada.}

Anderson, C., Rozzi, R., Torres-Mura, J.C., McGehee, S., Sherriffs, M.F., Schuttler, E., Rosemond, A.D. 2006. Exotic vertebrate fauna in the remote and pristine subAntarctic Cape Horn Archipelago, Chile. Biodiversity and Conservation. 15: 3295-3313.

Apaza, M., Valqui, J., Mangel, J., Roca, M., Alfaro, J., Santillan, L., Perret, J.P., Onton, G., Castaneda, C., Munemura, G. \& Tovar, A. 2004. Estado de Conservación de Lontra felina (Molina, 1782) en la Costa Peruana. Reporte para la Comisión Permanente del Pacífico Sur, Lima.

Arias-Schreiber, M. 1993. Interacciones entre lobos marinos Otaria byronia y la pesquería artesanal en el puerto de San Juan de Marcona, Perú. Tesis de Bachiller, Universidad Nacional Agraria La Molina, Lima, Perú.

Brack, A. 1978. Situación actual de las nutrias (Lutrinae, Mustelidae) en el Perú. En: Duplaix, N. (Ed.) Otters Proceedings of the First Working Meeting of the IUCN/SSC Otter Specialist Group. Paramaribo, Suriname. 76-84.

Cassini, M.H. 2008. Present status of Lontra felina in Argentina. Endangered Species Update. 25 (2): 57-60.

Castilla, J. \& Bahamondes, I. 1979. Observaciones conductuales y ecológicas sobre Lutra felina (Molina) 1782 (Carnívora: Mustelidae) en las zonas Central y Centro-Norte de Chile. Archivos de Biología y Medicina Experimentales. 12: 119-132.

Chéhebar, C. 1990. Action plan for Latin American otters. Otters: An action plan for their conservation. P. FosterTurley, P. S. MacDonald and C. Mason. Brookfield, Illinois, Chicago Zoological Society. 64-73.

Grimm, A., Barros, V., Doyle, M.E. 2000 Climate Variability in Southern South America Associated with El Niño and La Niña Events. Journal of Climate. 13: 3558.

Instituto Nacional de Estadisticas - Chile (INE). 2011. Compendio Estadístico Año 2011. Santiago. http://www.ine.cl/.

Instituto Nacional de Estadística e Informática - Perú (INEI). 2007. Resumen de Censos realizados entre 1940 y 2007. Lima. http://www.inei.gob.pe/.

IUCN, 2011. IUCN Red List of Threatened Species. Version 2011.1. http://iucnredlist.org. Accedida el 16 de mayo del 2011.

Larivière, S. 1998. Lontra felina. Mammalian Species 575: 1- 5.

Luebert, F. \& Pliscoff, P. 2006. Sinopsis bioclimática y vegetacional de Chile. Editorial Universitaria. Santiago de Chile.

Majluf, P., Babock, E., Riveros J.C., Arias, M. \& Alderete, W. 2002. Catch and Bycathc of Sea Birds and Marine Mammals in the Small-Scale Fishery of Punta San Juan, Peru. Conservation Biology 16 (5): 1333-1343. 
Mangel, J. \& Alfaro-Shigueto, J. 2008. Comunidades pesqueras y la conservación de la nutria marina (Lontra felina) en el sur de Perú. Revista Conservación Regional 2:5-10.

Mangel J.C., Whitty, T., Medina-Vogel, G., AlfaroShigueto, J., Caceres, C. \& Godley, B.J. 2011. Latitudinal variation in diet and patterns of human interaction in the marine otter. Marine Mammal Science 27 (2): E14-E25.

Medina-Vogel, G., Bartheld, J.L., Alvarez, R. \& Delgado, C. 2006. Population assesment and habitat use of marine otter (Lontra felina) in southern Chile. Wildlife Biology. 12 (2): 191-199.

Medina-Vogel, G., Merino, L.O., Monsalve Alarcón, R. \& Vianna, J. de A. 2008. Coastal-marine discontinuities, critical patch size and isolation: implications for marine otter conservation. Animal Conservation. 11: 57-64.

Sánchez, R. \& Ayala, L. 2006. Evaluacion de la distribución y abundancia del gato marino Lontra felina en la costa central y norte del Perú durante el otoño del 2006. Reporte APECO.

Santillán, L. \& Caro, K. 2007. Mamíferos Marinos en el Puerto de Salaverry y Zonas Adyacentes. Libro de resúmenes CONCIMAR 2007.

Schweigger, E. 1964. El litoral peruano. 2da edición. Universidad Nacional Federico Villareal.

Sielfeld, W. 1997. Las áreas protegidas de la XII Región de Chile en la perspectiva de los mamíferos marinos. Estudios Oceanológicos. 16: 87-107.

Sielfeld, W. K., \& Castilla, J.C. 1999. Estado de conservación y conocimiento de las nutrias en Chile. Estudios Oceanológicos. 18: 69-79.
Thiel, M., Bravo, M., Hinojosa, I.A., Luna, G., Miranda, L., Núñez, P., Pacheco, A.S. \& Vásquez, N. 2010. Anthropogenic litter in the SE Pacific: An overview of the problem and possible solutions. Journal of Integrated Coastal Zone Management. 11 (1):115-134.

Ulloa, R., Zambrano, M. \& Chevarria, A. 2010. Expediente Tecnico 'Area de conservación regional Morro SamaQuebrada de Burros’ Tacna, Perú.

Valqui, J., Hartl G.B. \& Zachos, F.E. 2010. Non-invasive genetic analysis reveals high levels of mtDNA variability in the endangered South-American marine otter (Lontra felina). Conservation Genetics. 11: 20672072.

Valqui, J., 2011. The marine otter Lontra felina (Molina, 1782): A review of its present status and implications for future conservation. Mammalian Biology. DOI: 10.1016/j.mambio.2011.08.004.

Vianna, J., Ayerdi, P., Medina-Vogel, G., Mangel, J.C., Zeballos, H., Apaza, M. \& Faugeron, S. 2010. Phylogeography of the Marine Otter (Lontra felina): Historical and Contemporary Factors Determining Its Distribution. Journal of Heredity. 101 (6): 676-689.

White, P.C.L., Jennings, N.V., Renwick, A.R. \& Barker, N.H.L. 2005. Questionnaires in ecology: a review of past use and recommendations for best practice. Journal of Applied Ecology. 42: 421-430.

\footnotetext{
${ }^{1}$ ProDelphinus. Enrique Palacios 630-204, Lima 18, Perú

${ }^{2}$ University of Exeter, Penyrn, Cornwall, TR10 9EZ, United Kingdom.

${ }^{3}$ Centro de Ornitología y Biodiversidad (CORBIDI). Calle Santa Rita 105 Of. 202, Lima 33, Perú.

${ }^{4}$ Instituto de Zoología, Universidad de Kiel. Am Botanischen Garten 1-9, 24118 Kiel, Alemania. Correo electrónico: jvalqui@zoologie.uni-kiel.de
} 